\title{
A METHOD FOR EXTRACTING SUBSTATION EQUIPMENT BASED ON UAV LASER SCANNING POINT CLOUDS
}

\author{
Wenhui $\mathrm{Yu}^{1}$, Jianfei $\mathrm{Xi}^{2}$, Zhengrong $\mathrm{Wu}^{1}$, Weigang Lei ${ }^{2}$, Changyu Zhu ${ }^{2}$, Tao Tang ${ }^{3, *}$ \\ ${ }^{1}$ China Southern Power Grid Company Limited, 510700 Guangzhou, China - (yuwh, wuzr)@ csg.cn \\ ${ }^{2}$ China Southern Power Grid Digital Grid Research Institute Co. Ltd, Guangzhou, China- (xijf, leiwg, zhucy)@ csg.cn \\ ${ }^{3}$ Wuhan Dynspai Technology Company Limited, Wuhan, China - dowtowne@ dynspai.com
}

\section{Commission II, WG II/3}

KEY WORDS: UAV; Laser Scanning; Point Clouds; Substation Extraction; Dimension Feature

\begin{abstract}
:
Smart grid construction puts higher demands on the construction of 3D models of substations. However, duo to the complex and diverse structures of substation facilities, it is still a challenge to extract the fine three-dimensional structure of the substation facilities from the massive laser point clouds. To solve this problem, this paper proposes a method for extracting substation equipment from laser scanning point clouds. Firstly, in order to improve the processing efficiency and reduce the noises, the regular voxel grid sampling method is used to down-sample the input point cloud. Furthermore, the multi-scale morphological filtering algorithm is used to segment the point cloud into ground points and non-ground points. Based on the non-ground point cloud data, the substation region is extracted using plane detection in point clouds. Then, for the filtered substation point cloud data, a three-dimensional polygon prism segmentation algorithm based on point dimension feature is proposed to extract the substation equipment. Finally, the substation LiDAR point cloud data collected by the UAV laser scanning system is used to verify the algorithm, and the qualitative and quantitative comparison analysis between the detected results and the manually extracted results are carried out. The experimental results show that the proposed method can accurately extract the substation equipment structure from the laser point cloud data. The results are consistent with the manually extracted results, which demonstrate the great potential of the proposed method in substation extraction and power system 3D modelling applications.
\end{abstract}

\section{INTRODUCTION}

In the digital era, smart grid construction puts forward higher demands on substation engineering construction (Guo et al., 2013; Zhang et al., 2013). Through the innovation of technical means, it is a must to further improve the construction and management level of substation engineering. At present, the main direction of China's power grid construction and development is to ensure the construction quality, improve the operation efficiency and reduce the construction cost.

Digital power grid is the data source of intelligent analysis and management for power grid, and it is the cornerstone of building smart grid. The key of digital power grid lies in the digital modelling and storage of pivotal components such as power grid facilities and so on. The results of digital modelling will become the basic data of the whole life cycle digital system of the project. Through the development of corresponding digital modelling and design standards, and with the help of 3D digital design means, the digital achievements of new power grid project can be transferred. However, for the existing power grid, it is faced with many problems, such as outdated or incomplete design data, nonstandard construction earlier, which leads to the inconsistency between the design and the final construction results. Therefore, it is necessary to use efficient three-dimensional model acquisition means to collect the actual construction scene data for accurate as-built model building, thus to provide the digital data basis for the construction of the smart grid (Tang et al., 2009, Lv et al., 2016).

\section{RELATED WORKS}

Laser scanning equipment can achieve 3D high-precision point cloud scanning of electrical equipment, and directly obtain highprecision $3 \mathrm{D}$ data of substations without affecting the safe operation of substations. The laser point cloud data with precise geometry description can also be fused with RGB or multispectral camera data. In addition to obtaining 3D geometric information, the material and texture information of the equipment under scanning can be obtained. Compared with the traditional modelling methods, 3D fine modelling using laser point cloud data fusion of multi-source spectral image data has the advantages of higher accuracy and efficiency, and lower labour cost and so on (Guan et al., 2016; Liu et al., 2010). 3D laser scanning measurement technology has important applications in the extraction of power grid equipment (Cheng et al., 2014).

The substation is an important part of the power grid, but compared with other ground objects (such as buildings, roads, etc.), the structure of substation facilities is more complex and the types are more diverse. How to extract the fine threedimensional structure of substation facilities from massive laser point cloud data is still an important problem to be solved in terms of the construction of digital power grid. At present, the target extraction of power equipment based on laser point cloud data mainly focuses on power grid components with simple geometry such as tower and power line. For example, Chen et al. (2015), and Lin et al. (2016) used 3D laser point cloud data obtained from UAV and airborne platform to extract power lines and conducted diagnostic analysis on safe distance of transmission channel; Peng. et al. (2017) extracted tower location

\footnotetext{
* Corresponding author
} 
from UAV point cloud data and established three-dimensional models of tower. However, compared with other targets (such as transmission lines, towers, buildings, roads, etc.), the structure of substation facilities is more complex and the types are more diverse. How to extract the fine three-dimensional structure of substation facilities from massive laser point cloud data is an important problem in digital power grid engineering construction. The research on extracting substation equipment from laser point cloud data is relatively rare. Li et al. (2016) proposed a 3D modelling method for substations based on massive point cloud data. In this method, the filtered point cloud is divided into different priority point cloud sets through point cloud data clustering, and then local surface fitting is performed for different point cloud sets respectively. Finally, different fitting surfaces are integrated to generate the final threedimensional model. This method can deal with massive point cloud quickly after clustering segmentation, but it requires complex geometric and topological relationship construction to ensure the integrity of local fitting surface and the merging rules between different surfaces. Peng et al. (2017) proposed a division method based on spatial region complexity for fine measurement of substations, and then used automatic and manual methods for point cloud de-noising, filtering and 3D visualization. Tian et al (2018) used Streetview panoramic camera and IMS3D mobile mapping system to collect 3D laser point cloud of substations, and then used 3D Max 3D modelling software to realize 3D model of substations. Based on the manually labelled training samples, Fang et al. (2015) used random forest to segment the substation point cloud and extracted the geometric structure of the substation point cloud.

Due to the large number of internal components and complex scene, there are many special-shaped structures and power components in the substation. The shape of the internal parts of the substation is irregular, and the structure of the feature points, characteristic lines and feature planes are complex and diverse. At present, the 3D modelling technology of the substation still needs large manual interaction, and it is still a difficulty to automatically extract the three-dimensional structure of the substation from the Massive point cloud (Fang et al., 2015; Peng et al., 2018). Therefore, this paper proposes a new method to extract the equipment and facilities from the Massive point cloud data.

\section{EQUIPMENT EXTRACTION BASED ON LIDAR POINT CLOUD DATA IN SUBSTATION}

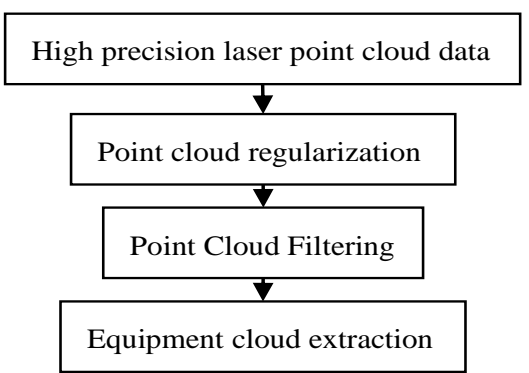

Figure.1 Overall workflow of the method

The whole algorithm can be divided into the following parts, and the work flow is shown in Figure 1. 1) Regularization of point cloud data. Aiming to solve the problems of large redundancy, long reconstruction time and low efficiency of $3 \mathrm{D}$ point cloud data, a point cloud simplification algorithm based on regular voxelized mesh down sampling is adopted for subsequent processing. 2) Point clouds separation. The original point cloud data contains a large number of ground data. The ground and the non-ground data must be separated. In this paper, morphological filtering is used for the separation. The non-ground data are used for further extraction. 3) Point cloud extraction of the substation equipment. This paper designs a method of extracting substation area based on the consistency estimation of point cloud normal to filter out the irrelevant non-surface point data outside the substation area. Based on the linear characteristics of substation equipment model, the point cloud dimension features are calculated to extract the fine equipment structure.

\subsection{Regularization of Substation Point Clouds}

In order to improve the processing efficiency, it is necessary to simplify the point cloud and eliminate the redundancy in the point cloud data. This paper adopts a simplified point cloud processing method based on regular voxels. The point cloud data is compressed by regular voxel down-sampling, and the spatial shape and structure features of the point cloud are saved at the same time, which improves the efficiency of subsequent point cloud filtering and feature calculation.

By calculating the side length $\mathrm{L}$ of the cube grid, the original point cloud is decomposed into $m \times n \times l$ small grids. The $\mathrm{X}\left(\mathrm{X}_{\min }, \mathrm{X}_{\max }\right), \mathrm{Y}\left(\mathrm{Y}_{\min }, \mathrm{Y}_{\max }\right), \mathrm{Z}\left(\mathrm{Z}_{\min }, \mathrm{Z}_{\max }\right)$ values are obtained by traversing all point clouds, and the length of three sides of the 3D voxel grid are calculated according to formula (1). In order to ensure that the laser point points are in the threedimensional bounding box, the bounding box are extended outward, and the distance correction of $\lambda$ is increased, which is usually set as the side length of single voxel.

$$
\begin{aligned}
& L_{X}=\left(X_{\text {max }}-X_{\text {min }}\right)+\lambda \\
& L_{X}=\left(Y_{\text {max }}-Y_{\text {min }}\right)+\lambda \\
& L_{X}=\left(Z_{\text {max }}-Z_{\text {min }}\right)+\lambda
\end{aligned}
$$

The minimum 3D bounding box $\operatorname{Cube}\left(\mathrm{L}_{\mathrm{x}}, \mathrm{L}_{\mathrm{y}}, \mathrm{L}_{\mathrm{z}}\right)$ are calculated for preparing regularized $3 \mathrm{D}$ point cloud data by the data statistics. According to the set edge length $V_{\text {length }}$, the minimum 3D bounding box space is divided into $\mathrm{m} \times \mathrm{n} \times \mathrm{l}$ voxels. Firstly, each laser point is assigned into the corresponding voxel; Secondly, the original point cloud is normalized by calculating the centre of mass $\mathrm{P}$ of each voxel and the point clouds in this voxel is replaced with P. After the voxel regularization, a large number of redundant points in the point cloud are eliminated.

\subsection{Separation of the point clouds}

The original substation point clouds contain a large number of ground points. Thus, it is necessary to separate the point clouds into ground points or non-ground points. In this paper, the morphological method (Zhang et al., 2003, Balado et al., 2020) is adopted to filter the point clouds. Two basic operations of expansion and corrosion are defined as equation 2,3 in morphology. (Zhang et al., 2016).

Corrosion:

Expansion:

$$
(f \otimes g)(i, j)=Z(i, j)=\min _{Z(s, t) \in w} Z_{0}(s, t)
$$

$$
(f \oplus g)(i, j)=Z(i, j)=\min _{Z(s, t) \in w} Z_{0}(s, t)
$$

Where $f$ is the image to be processed $\mathrm{g}$ is the morphological structure element

$\mathrm{Z}(\mathrm{i}, \mathrm{j})$ is the corresponding value of the $(\mathrm{i}, \mathrm{j})$ pixel position of the image after morphological operation 
$w$ is the structure element window

$\mathrm{Z}_{0}(\mathrm{~s}, \mathrm{t})$ is the value of pixel position $(\mathrm{s}, \mathrm{t})$ in the corresponding area of the structure element window in the original image.

When the morphological theory is applied to LiDAR point cloud data filtering, the size of structural elements is set larger than the size of ground objects, and carry out opening operation to filter out the surface objects. The mathematical expression is as follows:

$$
(f \circ g)=((f \otimes g) \oplus g)
$$

First, the structural element $g$ is used to corrode $f$, and then the corrosion result is expanded. The mutil-scale morphological is designed to segment the point clouds. To be specifically, the filtering window adopts a linear increasing gradient structure element according to the characteristics of different features. The detail process is as follows:

(1) The corrosion. Set the structure element window size to $\mathrm{w} \times \mathrm{w}$, and use this window to traverse the laser point clouds For each point in the structural window, the elevation distribution is calculated, and the minimum elevation in the window of the structure is taken as the elevation value after corrosion.

(2) The expansion. The structure element window is set to be the same size as in step $1(\mathrm{w} \times \mathrm{w})$. After traversing the laser point clouds, the data obtained after step 1 is processed by dilation operation with a $w \times w$ structure window. Firstly, the original point cloud elevation value is replaced with the output point cloud elevation value in step 1 . Then comparing with the elevation of each point in the structure window, and the elevation maximum value in the $\mathrm{w} \times \mathrm{w}$ window is taken as the expanded elevation value.

(3) Ground point extraction. Whether the point is a ground point or not can be determined by the elevation difference generated via the process of corrosion and expansion. Let $Z_{p}$ be the original elevation of point $\mathrm{p}$. After steps 1-2, the absolute value of the elevation difference between the expanded elevation value $\mathrm{Z}_{\mathrm{P} 1}$ of point $\mathrm{P}$ and the original elevation value $\mathrm{Z}_{\mathrm{P}}$ are calculated. If the absolute value of the elevation difference is less than or equal to the hard threshold $t$, then point $p$ is classified as a ground point. Otherwise, it will be classified as a non-ground point.

\subsection{Substation Equipment Extraction}

The substation equipment point clouds are encircled in the nonground point clouds after the morphological filter segmentation. The necessary step is to localize the cover range of the substation, that is, to calculate the convex polygon of the substation, which could be obtained based on the levelling property of the substation. To be specifically, the localization of the substation is based on an assumption that the ground plane of the substation is horizontal. By estimating the normal distribution of the ground point clouds, the plane $P_{\text {large }}$ that contains the largest number of points could be extracted. The convex polygon construction method, alpha-shape (Santos et al., 2019), is used to calculate the convex polygon boundary of the $P_{\text {large }}$. Appearently, the substation equipment's boundary is the same with the $P_{\text {large }}$ 's boundary. However, the convex boundary could only provide a rough range of substation. In order to extract the fine structure of substation, a dimension feature based method is designed to refine the substation equipment point clouds.
Point cloud dimension feature (Hackel et al., 2016, Weinmann et al. 2017) can describe the shape distribution of point cloud, and it has been widely used in point cloud segmentation and classification (Yang et al., 2013). And substation equipment usually has the characteristics of line and plane distribution. Planar devices (e.g. transformers) are usually connected to linear devices (e.g. conductors, insulators). Thus, in this paper, the dimension feature and region growth algorithms are combined to extract substation equipments. The dimension feature of LiDAR point cloud is mathematically defined as:

$$
\left(\mathrm{a}_{1 \mathrm{D}}, \mathrm{a}_{2 \mathrm{D}}, \mathrm{a}_{3 \mathrm{D}}\right)=\left(\frac{\sqrt{\lambda_{1}}-\sqrt{\lambda_{2}}}{\sqrt{\lambda_{1}}}, \frac{\sqrt{\lambda_{2}}-\sqrt{\lambda_{3}}}{\sqrt{\lambda_{1}}}, \frac{\sqrt{\lambda_{3}}}{\sqrt{\lambda_{1}}}\right)
$$

Where $\quad a_{1 D}+a_{2 D}+a_{3 d}=1$

$\lambda_{1}, \lambda_{2}, \lambda_{3},\left(\lambda_{1} \geq \lambda_{2} \geq \lambda_{3}\right) \quad$ are the matrix eigenvalues obtained from the covariance matrix constructed by the point cloud neighbourhood point set.

In order to determine the optimal size of the neighbourhood radius and avoid the inaccurate description of local geometric features of point clouds, this paper adopts the method of minimizing the dimension feature entropy function to realize the adaptive calculation of the neighbourhood size and select the optimal neighbourhood radius. The basic principles are as follows: Firstly, the minimum and maximum neighbourhood search radius and interval step size, $r_{\min }$ (initialized as $0.5 \mathrm{~m}$ ), $r_{\max }$ (initialized as $2 \mathrm{~m}$ ), $\mathrm{r}_{\text {step }}$ (initialized as $0.1 \mathrm{M}$ ) are fixed empirically; Secondly, matrix decomposition is conducted in a certain radius to calculate the eigenvalue $\lambda_{1}, \lambda_{2}, \lambda_{3}$ and it calculate corresponding $\left(a_{1 D}, a_{2 D}, a_{3 D}\right)$ value; Finally, entropy function is defined by equation 6 :

$$
E_{f}=-a_{1 D} \ln \left(a_{1 D}\right)-a_{2 D} \ln \left(a_{2 D}\right)-a_{3 D} \ln \left(a_{3 D}\right)
$$

Where $E_{f}$ is the entropy of the sum of dimension feature.

According to formula (6), radius that corresponds to the minimum value of $E_{f}$ is the optimal neighbourhood radius, and the obtained $\left(\mathrm{a}_{1 \mathrm{D}}, \mathrm{a}_{2 \mathrm{D}}, \mathrm{a}_{3 \mathrm{D}}\right)$ describes the feature description of point cloud geometry distribution.

Mathematically, for a linear target's point cloud, the eigenvalue of the neighbourhood point set in its own principal direction is much larger than that in the other two directions, namely the eigenvalue $\lambda_{1} \gg \lambda_{2} \cong \lambda_{3}$. Thus, the point clouds of linear equipment have good distinguishability, leading them to be selected as the seed points for region growth, in which the points set with the same linear distribution dimension characteristics are generated. Through the spatial connection of different devices, linear and planar equipment could be extracted, so as to realize the extraction of structured substation facilities.

\section{EXPERIMENTAL}

The whole approach proposed in this paper is implemented by $\mathrm{C}++$ and PCL open source library under Windows 10 . The point clouds of a typical substation collected by UAV LiDAR system is used to verify the method. The data acquisition and sensor calibration is not the main topic studied in this paper. Thus the data quality is assumed to full fill the demand. The data coverage of the laser point cloud substation is $480 \mathrm{~m} \times 370 \mathrm{~m}$. The average point density is 17 points $/ \mathrm{m}^{2}$, a total of 4574697 points. The original LiDAR point cloud data is shown in Figure 2 (a) (b). 


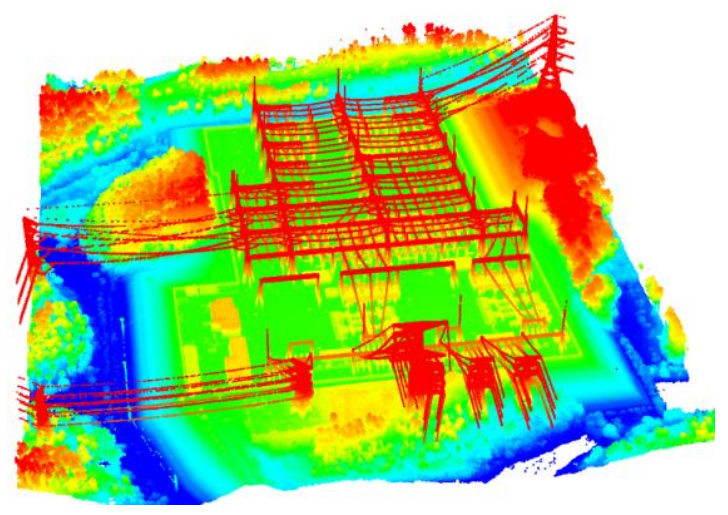

(a) Overview snapshot of the data

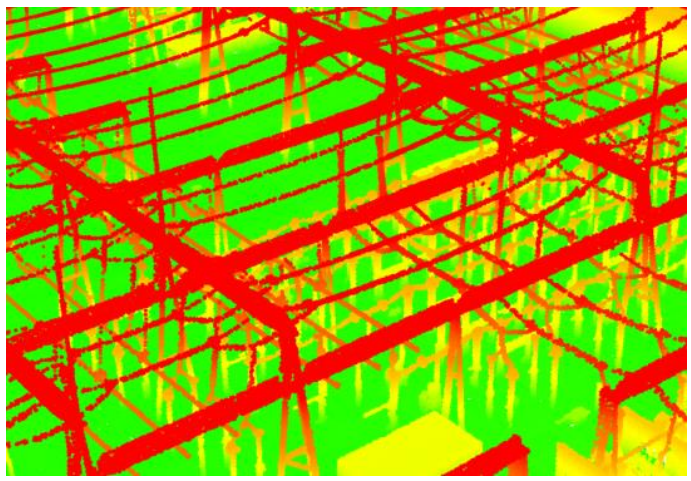

(b) Detailed snapshot of the experimental data

Figure 2. Overview and detailed snapshot of the data

Figure 3 shows the overall and details snapshot of the point clouds before and after regularization. The point clouds are rendered with height, and the green represents the higher height. Through the regularized point cloud details, as shown in Figure 3 (c) (d), it can be speculated that after voxel regularization, the phenomenon of uneven point density and uneven scanning line spacing in the original point cloud has been eliminated. It avoids the influence of uneven point density and spacing, reducing the robustness of subsequent equipment extraction.

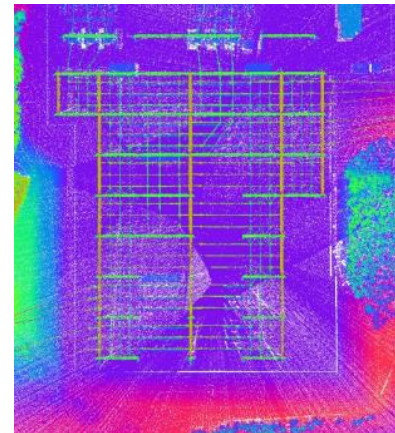

(a) Before the data regularization

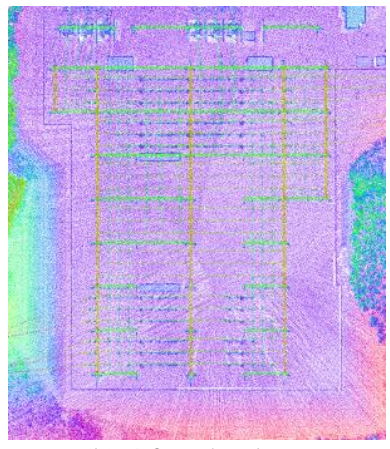

(b) After the data regularization

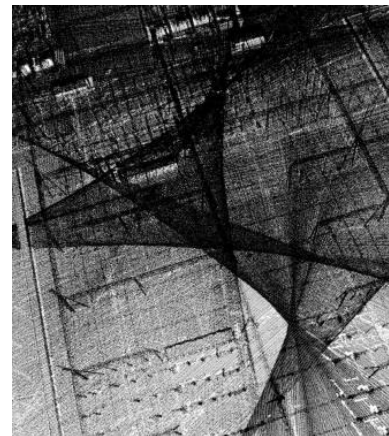

(c) Detailed before regularization

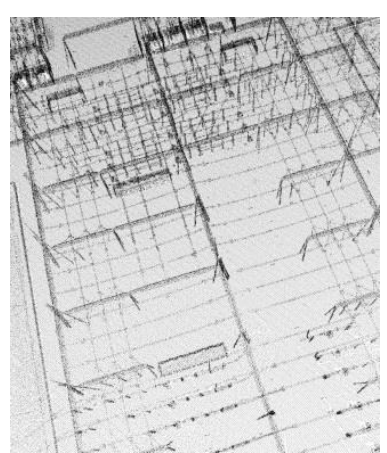

(d) Detailed after regularization
Figure 3. Overview and detailed snapshot of the experiment data before and after the data regulation, and the (c)(d) are rendered with grey to better show the uneven density.

The morphological filtered results are shown in Figure 4. Figure 4 (a) is the extracted substation ground point cloud data, mainly including the substation area ground point cloud (flat area) and surrounding undulating terrain. Figure 4 (b) shows the extracted non-ground point cloud data, including substation equipment point cloud data and substation outside non-ground point cloud data. The non-ground point cloud outside the substation is mainly composed of vegetation and trees. Compared with Figure 4 (a) (b), it can be seen that after morphological filtering, the ground point cloud data is well filtered. But due to the interference of the surrounding non-substation point clouds, there are a large number of non-equipment point clouds in the extracted nonground points. Therefore, this paper designs a method for extracting substation area based on the consistency estimation of point cloud normal, in which the irrelevant non-ground point data outside the substation area are filtered out.

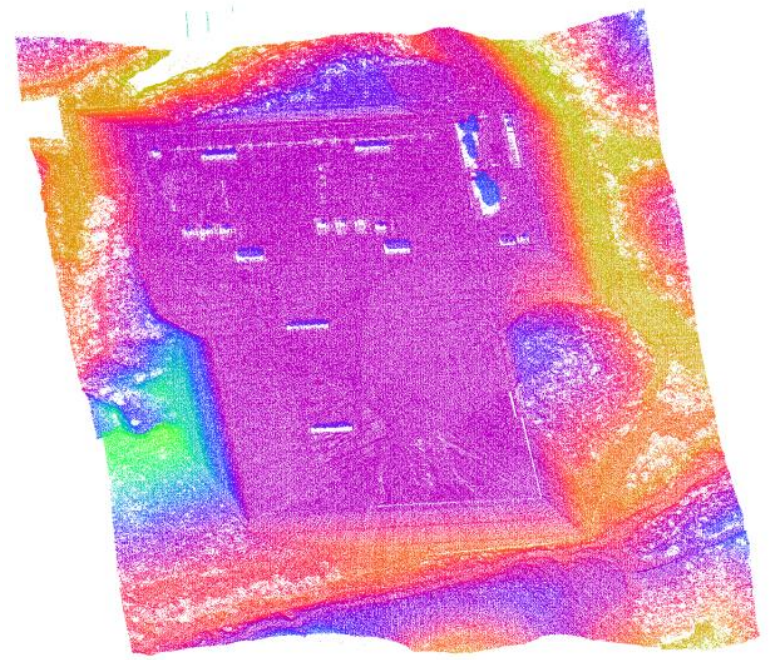

(a) Extracted ground points. The ground points mainly contain the levelling area point clouds and the terrain points. 


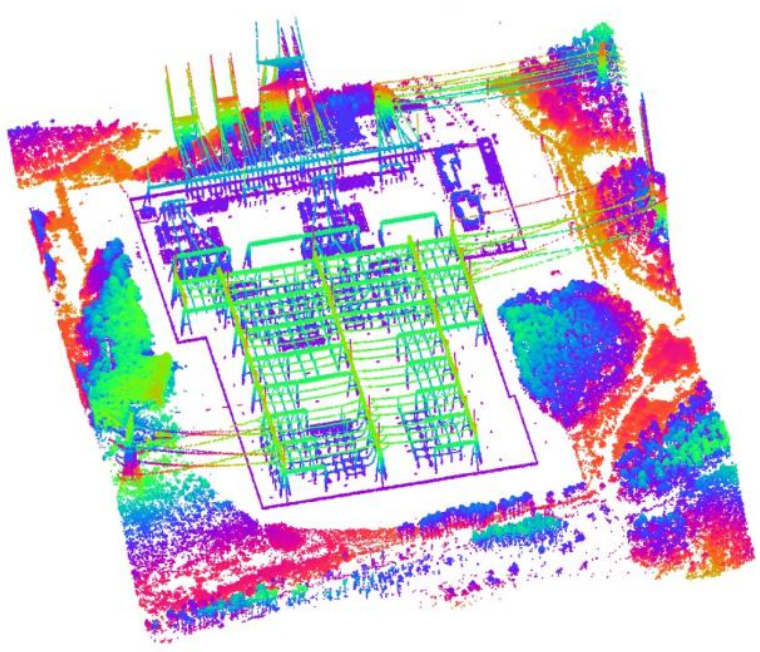

(b) Extracted non-ground points. The non-ground points mainly contain the substation equipment points and the vegetation points

Figure 4. Morphological filtering of the point clouds

Figure 5 shows the result of substation area extraction based on point cloud normal consistency estimation. Figure 5 (a) is the top view of the extracted ground point cloud data of the substation area. Through the constraint of normal vector in vertical direction, the irrelevant point cloud data outside the substation area are filtered out. Figure 5 (b) is a side view of the extracted results. It can be seen that the extracted results have good consistency in the elevation direction, which conforms to the flat characteristics of the substation area.

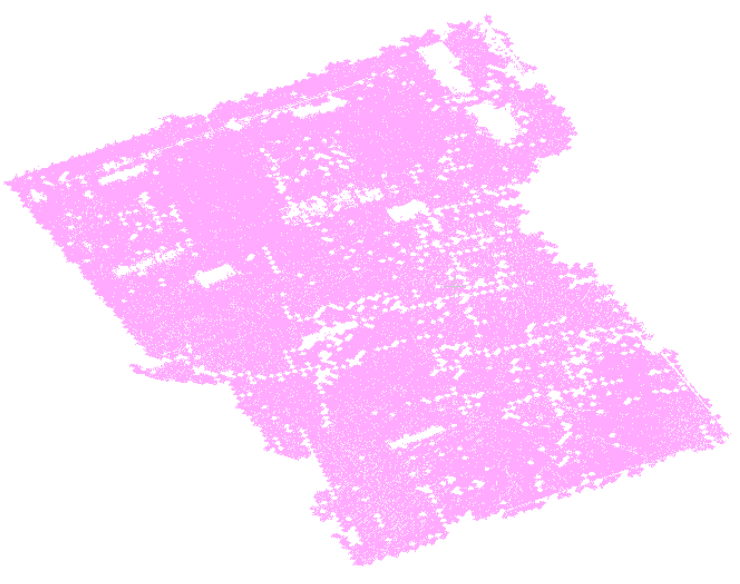

(a) Substation levelling area extraction results.

\section{(b) Side view of point cloud}

Figure 5. Extraction of the substation region from the point clouds. (a) shows that the normal constraint in vertical direction is a powerful way to filter out the non-equipment point clouds. The point clouds are rendered with height value, And the color consistent also shows that the assumption of substation levelling area. (b) demonstrates that the assumption is reasonable. The convex boundary of levelling area of substation is consistent with the equipment's boundary

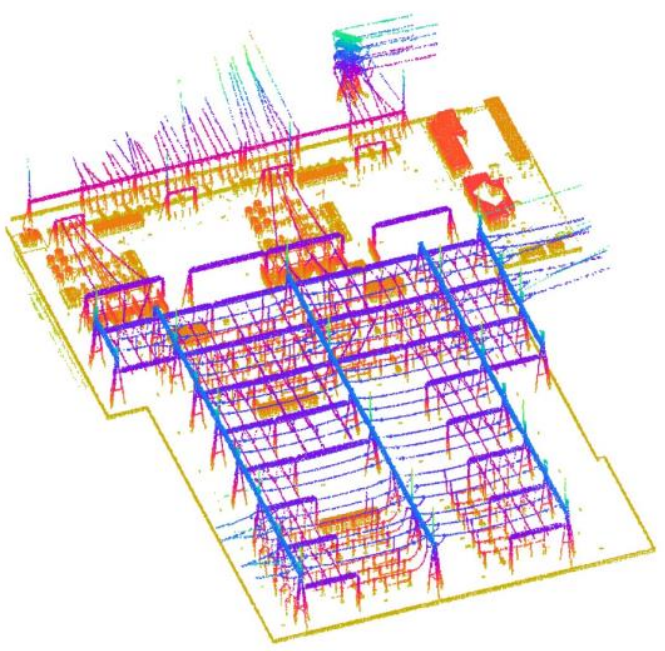

Figure 6. Extraction of the equipment point clouds after dimension feature estimation and region growth. The main body of substation point clouds are maintained, which is a foundational process for substation reconstruction.

After the substation area is located, the point cloud data in the substation area are classified based on the dimension feature. By calculating the dimension feature of the point clouds pointwisely, the point clouds with linear and plane feature are retained to form the point clouds of substation equipment, as shown in Figure 6. Compared with Figure 5 (b), the point cloud data outside the substation area in the non-ground points and the point cloud data of non-substation equipment inside the substation have been well filtered, and the equipment point cloud in the substation has been well preserved, thus laying a good data foundation for the subsequent modelling and analysis.

In the quantitative comparative analysis, the manually selected power substation equipment point clouds are taken as the ground truth of the substation equipment. And the results extracted by the method proposed in this paper are compared with the ground truth. At the same time, the time costs in each step of the algorithm are calculated. The experimental results are shown in Table 1. According to statistics, the time consumption of the three stages of point clouds regularization, point clouds filtering and substation equipment extraction are $48 \mathrm{~s}, 92 \mathrm{~s}$ and $50 \mathrm{~s}$ respectively. And additionally, the substation levelling area localization's time consuming is $43 \mathrm{~s}$ The extraction accuracy and recall rate of point clouds filtering, substation levelling area localization and substation equipment extraction are $72 \%$ / 90\%, $91 \% / 81 \%$ and $83 \% / 84 \%$ respectively. The point clouds distance between the automatically extracted and the manually extracted is almost smaller than $0.1 \mathrm{~m}$, which is accurate enough for the following fine modelling.

At the same time, the efficiency of this algorithm is compared with that of manual extraction. The substation extraction time per square metre of each two different extraction process are treated as the efficiency metric. The smaller time, the higher efficiency. The efficiency of this algorithm is $7 \mathrm{~s} / \mathrm{m}^{2}$, and that of manual algorithm is $87 \mathrm{~s} / \mathrm{m}^{2}$. The extraction efficiency of this algorithm is about 12.4 times higher than that of manual algorithm, which greatly improves the extraction efficiency of equipment laser point clouds in substation. 


\begin{tabular}{|c|c|c|c|}
\hline Algorithm steps & $\begin{array}{c}\text { Time } \\
\text { consuming (s) }\end{array}$ & $\begin{array}{c}\text { Extraction } \\
\text { accuracy (\%) }\end{array}$ & $\begin{array}{c}\text { Recall } \\
(\%)\end{array}$ \\
\hline $\begin{array}{c}\text { Regularization of } \\
\text { point cloud data }\end{array}$ & 48 & -- & -- \\
$\begin{array}{c}\text { Point Cloud Filtering } \\
\text { Substation area } \\
\text { extraction }\end{array}$ & 92 & $72 \%$ & $90 \%$ \\
$\begin{array}{c}\text { Substation equipment } \\
\text { extraction }\end{array}$ & 50 & $91 \%$ & $81 \%$ \\
\hline
\end{tabular}

Table 1. The time efficiency in each step of extraction

\begin{tabular}{|c|c|}
\hline Extraction method & $\begin{array}{c}\text { Substation equipment } \\
\text { extraction }\left(\mathrm{s} / \mathrm{m}^{2}\right)\end{array}$ \\
\hline Manual extraction & 87 \\
The algorithm in this paper & 7 \\
\hline
\end{tabular}

Table 2. Efficiency comparison between automatic extraction and manual extraction

\section{CONCLUSION}

The extraction of equipment laser point clouds in substation is the key and foundation of analysing the pivotal size of substation equipment and auxiliarily modelling equipment entity. This paper proposes a method for extracting equipment point clouds from UAV point clouds. And the detection results are evaluated qualitatively and quantitatively from point clouds collected by UAV LiDAR system. The accuracy of the proposed method is about equal to the traditional manual extraction method. efficiency. The experimental results show that the proposed method can significantly improve the efficiency by 12 times in this test area. That also shows the automation of this work. Future work will focus on the automatic construction of entity model under fine scale for the extracted substation facility data.

\section{ACKNOWLEDGEMENTS}

Thanks for the support of Science and technology project of China Southern Power Grid Company Limited (ZBKJXM20170229)!

\section{REFERENCES}

Balado, J., Oosterom, P. van, Díaz-Vilariño, L., Meijers, M. 2020. Mathematical morphology directly applied to point cloud data. ISPRS Journal of Photogrammetry and Remote Sensing, 168, 208-220. doi.org/10.1016/j.isprsjprs.2020.08.011.

Chen, C., Mai, X., Song, S., Peng, X., Xu, W., Wang, K., 2015. Automatic power lines extraction method from airborne LiDAR point cloud. Geomatics and Information Science of Wuhan University, 40(12), 1600-1605. doi.org/10.13203/j. whugis20130 573.

Cheng, L., Tong, L., Wang, Y., Li, M., 2014. Extraction of urban power lines from vehicle-borne LiDAR data. Remote Sensing. doi.org/10.3390/rs6043302.

Fang, Y. J., Tang, M., Luo, J., Pang, Z. Q., 2015. Automatic point clouds segmentation for substation 3D modeling based on random forest algorithm. Manufacturing Automation(10), 86-89.

Guan, H., Yu, Y., Ji, Z., Zhang, Q., 2016. Extraction of powertransmission lines from vehicle-borne lidar data. International Journal of Remote Sensing, 37(1), 229-247. doi.org/10.1080/014 31161.2015.1125549.
Guo, W., 2013. Application status and development of smart grid technology. Power Supply Technologies and Applications(05), 273. doi.org/CNKI:SUN:DJYY.0.2013-05-2 25.

Hackel, T., Wegner, J.D., Schindler, K. 2016. Contour detection in unstructured $3 \mathrm{~d}$ point clouds. IEEE Conference on Computer Vision and Pattern Recognition, 1610-1618. doi.org/10.1109/ CVPR.2016.178.

Li, J., Wang, B., Wang, X., Zhang, Y., Xu, Z., 2016. Full 3D modeling of transformer station based on massive point cloud. North China Electric Power(07), 26-30. doi.org/10.16308/j.cnki. issn1003-9171.2016.07.005.

Lin, X., Duan, M., Zhang, J., Yi, Z., 2016. A method of reconstructing $3 \mathrm{D}$ powerlines from airborne LiDAR point clouds. Science of Surveying Mapping. doi.org/10.16251/j. cnki.1009-2307.2016.01.021.

Liu, Q. L., Hu, W. S., Wang, C., Xu, J. M., 2010. Realization of the 3D simulation model based on laser scanner. Egineering of Surveying And Mapping.(5), 43-45. doi.org/10.3969/j.issn.10067949.2010.05.010.

Lv, L., Shan, B., Yan, S., 2016. 3D digital power network construction based on laser scanning technology. Journal of Geomatics, 041(3), P.93-96. doi.org/10.14188/j.20956045.2016. 03.023.

Peng, H., Xu, J., Ni, S., 2017. Application of ground 3D laser scanning technique in substation fine measurement. Bulletin of Surveying and Mapping, 000(12), 107-111. doi.org/10.13474/j. cnki.11-2246.2017.0390.

Peng, L., Huang, Z., Cheng, P., 2018. Three-dimensional visualization of substation based on 3D laser point cloud data. Geotechnical Investigation Surveying, 046(4), 65-67.

Peng, X., Song, S., Qian, J., Chen, C., Wang, K., Yang, Y., Zheng, X., 2017. Research on automatic positioning algorithm of power transmission towers based on UAV LiDAR. Power System Technology, 41(11), 3670-3677. doi.org/10.13335/j.1000-3673. pst.2016.3268.

Santos, R.C., Galo, M., Carrilho, A. 2019. Extraction of building roof boundaries from lidar data using an adaptive alpha-shape algorithm. IEEE Geoscience and Remote Sensing Letters, 16, 1289-1293.

Tang, Y., Shao, Z., Guo, C., Cao, Y., Ruan, Q., 2009. Digital power grid architecture. (6), 115-118. doi.org/10.3969/j.issn. 1006-6047.2009.06.026.

Tian, X., Zhao, F., Liu, H., Zeng, Q., Liu, L., 2018. Realization and prospect of $3 \mathrm{~d}$ simulation model of substation based on point cloud data. Sichuan Electric Power Technology, v.41; No.256(04), 36-40. doi.org/CNKI:SUN:SCDL.0.2018-04-009.

Weinmann, M., Weinmann, M. 2018. Geospatial computer vision based on multi-modal data - how valuable is shape information for the extraction of semantic information? Remote Sensing, 10, 2 .

Yang, B., Zhen, D., 2013. A shape-based segmentation method for mobile laser scanning point clouds. ISPRS Journal of Photogrammetry Remote Sensing, 81(7), 19-30. doi.org/10. 1016/ j.isprsjprs.2013.04.002. 
Zhang, W., Qi, J., Peng, W., Wang, H., Xie, D., Wang, X., Yan, G., 2016. An easy-to-use airborne lidar data filtering method based on cloth simulation. Remote Sensing, 8(6), 501. doi.org/ $10.3390 / \mathrm{rs} 8060501$.

Zhang, K., Chen, S. C., Whitman, D., Shyu, M. L., Yan, J., Zhang, C. 2003. A progressive morphological filter for removing nonground measurements from airborne LIDAR data. IEEE Transactions on Geoscience and Remote Sensing, 41(4), 872882. doi.org/10.1109/TGRS.2003.810682. 